\title{
PHASE-COHERENT INTERFEROMETRIC IMAGING
}

\author{
J.E. BALDWIN \\ Mullard Radio Astronomy Observatory \\ Madingley Rd., Cambridge CB3 OHE, U.K.
}

\section{Introduction: early history}

The essence of high resolution interferometric imaging is Michelson interferometry and it has a long history. Yet the symposium programme describes it as the Last Frontier! Why was it not crossed decades ago? This paradox needs some explanation. I suspect that many participants here are tourists in interferometry, as I am in their fields, so it is interesting to look at the key developments since Michelson invented the field. It is, of course, a personal view.

Michelson's paper on measurements of the diameters of the Galilean satellites of Jupiter (1891) contains most of the basic ideas. In particular it shows (Fig.1) the Fourier transform relationship between the visibility $\mathrm{V}$ of the fringes in a two-slit Michelson stellar interferometer and the distribution of brightness across the source $\phi(x)$ as a function of the separation of the slits. The only assumption is that each element of the source radiates incoherently. But notice that, instead of taking $V=($ cosine term $)+i$ (sine term) which would contain the phase of the complex visibility, he uses $V^{2}$. Why? Because he understood that the fluctuations in optical path through the atmosphere completely destroy the true astronomical phase of the fringes. Thus the direct Fourier transform reconstruction becomes impossible. So he made the assumption which would satisfy many readers, but not the attendees at this symposium, that all stars are essentially symmetrical. All interferometric phases would be either $0^{\circ}$ or $180^{\circ}$. He could then measure the diameters of Jupiter's moons with a small telescope. The natural step to extend this to measure stellar diameters took a further 30 years. The 20 foot stellar interferometer on the Mt.Wilson 100-inch telescope was a great success in 1920, providing measurements of the angular diameters of about 20 stars; ten years later, the 50 -foot free-standing version never fulfilled its promise. The instrumental problems of mechanical stability, short timescales of the atmospheric fluctuations and weak flux of light, still pro- 
NATURE [DECEMBER I7, I891

for producing such fringes, by providing the cap of the objective with two parallel slits, adjustable in width and distance apart. If such a combination be tocussed on a star, then, instead of the concentric rings before mentioned, there will be a series of straight equidistant bands whose length is parallel with the slits, the central one being brightest, ${ }^{2}$ Fig. $1, c$.

The general theory of these fringes may be found in the Philosophical Magazine for March i 91 . The general cquation showing the relation between the i'isibility of the fringes and the distance between the slits is:

$$
\mathrm{V}^{2}=\frac{\left[\int \phi(x) \cos k x d x\right]^{2}+\left[\int \phi(x) \sin k x d x\right]^{2}}{\left[\int \phi(x) d x\right]^{2}} .
$$

which reduces to the simpler form

$$
\mathrm{V}=\frac{\int \phi(x) \cos k x d x}{\int \phi(x) d x}
$$

when the object viewed is symmetrical.

Figure 1. Michelson on Measurement of Jupiter's Satellites by Interference.

vide the basic instrumental constraints in optical interferometry. It seems likely that it was this relative failure, together with the lack of fast and sensitive detectors other than the human eye, which halted further development at optical wavelengths for decades.

\section{Radio interferometry}

Meanwhile, radioastronomy emerged in the late 1940's and from the start was preoccupied with the need to attain higher angular resolution than any single antenna could provide. Progress was rapid. The first use of interferometry in 1946 was followed by the one-dimensional Fourier reconstruction of the brightness distribution across the Sun in 1950 and the first two-dimensional image incorporating phase measurements in 1952 (O'Brien (1953)). The technical problem of phase stability at radio wavelengths was not mechanical but electronic and, for radio-linked interferometers, atmospheric. A method for overcoming this, now known as closure phase, was invented by Jennison (1958). The idea is this: take three telescopes giving three interferometer baselines forming a closed triangle and measure the apparent fringe phases on all baselines simultaneously. Suppose that on the first baseline, between telescopes 1 and 2, the astronomical phase associated with the source is $\phi_{12}$ and there are atmospheric phase paths $\theta_{1}$ and $\theta_{2}$ above the two telescopes. Then the observed phase is $\left(\phi_{12}+\theta_{1}-\theta_{2}\right)$ The 
other baselines give similar expressions with cyclic changes in 1,2 and 3 . The closure phase is the sum of the observed phases on the three baselines, $\left(\phi_{12}+\phi_{23}+\phi_{31}\right)$, and is independent of the atmospheric terms. This elegant invention made almost no impact on the subject at the time!

The next few years saw instead the development of earth-rotation aperture synthesis by Ryle and Neville (1962) and the construction of many large arrays including the Cambridge One-Mile and 5-km Telescopes, the Westerbork array and the VLA, all based on that principle. The atmospheric irregularities presented an interesting but not an overwhelming challenge and correction methods could be developed as the baselines were increased and shorter wavelengths used. A limit was reached when very long baselines were introduced. It was at this point that the idea of closure phase was revived by Rogers et al.(1974) and applied to VLBI image reconstruction. It rapidly became the standard technique. What is suprising at first sight is that one can reconstruct images precisely with just the closure phases and not all the phase information. One physical reason is that the sources studied are not arbitrary mathematical functions but objects of strictly positive intensity separated on the sky by regions which are effectively black. No explicit analytical proof has been found that the images are unique but very exhaustive simulations have shown that images can be restored reliably from such data.

The remarkable result of all of these developments over a period of forty years is that both the sensitivity and the resolution of radio interferometric imaging using aperture synthesis have been improved by factors of roughly $10^{9}$. That experience is a strong incentive to pursue aperture synthesis at optical and infra-red wavelengths. The principles of electromagnetism are identical; it is simply a matter of overcoming the technical difficulties. Even a small fraction of that factor of $10^{9}$ improvement would transform conventional imaging. What is needed is a phase-coherent array of telescopes.

\section{Phase-coherent optical arrays}

The revival of interest in Michelson interferometry was pioneered largely by Labeyrie (1975) and was followed over the next ten years by the construction of several interferometers including the I2T and GI2T in France, the Mt.Wilson stellar interferometer in the U.S.A. and the Sydney prototype interferometer in Australia. These established many of the technologies necessary for reliable interferometric measurements, but all were designed to operate with only two telescopes at any one time. Thus they could not provide absolute phase information or be used for imaging.

No experimental work on optical closure phase measurement had been done by the mid-1980's, 25 years after its first proposal. It was suprising 


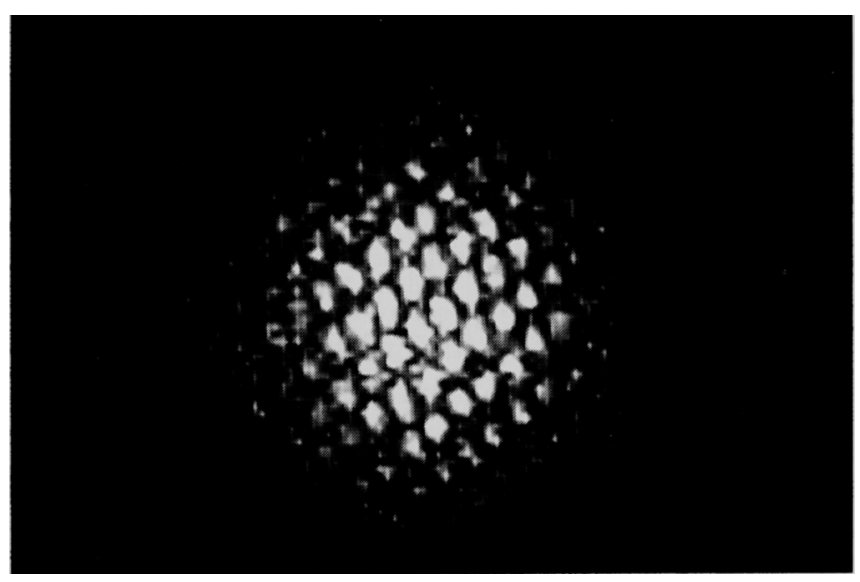

Figure 2. Image of Vega using a 3-hole aperture mask on the University of Hawaii 88-inch Telescope.

to find that a very simple experiment was waiting to be done. If a mask with three small apertures is placed at a reimaged pupil in a telescope, or indeed almost anywhere in its optical train, the image of a star becomes the Airy disk corresponding to the size of the individual apertures crossed by three sets of interference fringes (see Fig.2). These correspond to the three baselines between the three apertures. The closure phase can be calculated by taking an arbitrary origin in the pattern and summing the phase displacements of the three sets of fringes relative to this point. The result is independent of the point chosen. The effect of atmospheric changes in path above the three apertures is to move the fringe pattern laterally, but not to change its shape. The closure phase is an intrinsic feature of the pattern. In this case, for an unresolved star, the maxima of all three fringe patterns superpose at a grid of positions and the closure phase is zero.

The success of this experiment (Baldwin et al. (1986)) then led us to imaging experiments using non-redundant aperture masks on the pupils of large telescopes. The aims were twofold. First, to achieve diffraction-limited performance from ground-based telescopes and to use that for studies of the brightest red giants and super-giants, the only stars resolvable by existing $4 \mathrm{~m}$ telescopes. The second aim was to establish the techniques for imaging arrays in a particularly simple environment. Since telescope optics ensure that all rays arriving at a point on the image have traversed equal optical paths, there is no need to employ the precise, variable path delay systems which are necessary when attempting interference between separate telescopes. The motivation for using non-redundant aperture masks, in which no baseline is repeated, is that each short exposure image gives an estimate 
of the closure phases which is unbiased by atmospheric variations. The use of one-dimensional arrays allows all the fringe information to be encoded in one dimension on a CCD detector, thereby enabling both short (a few ms) exposure and readout times for the data. Sufficient data can be recorded in about 5 minutes to give good signal-to-noise ratios on several baselines for most stars of interest.

Observations by Buscher et al. (1990) using the Wlliam Herschel $4.2 \mathrm{~m}$ telescope demonstrated the existence of a hot-spot on the surface of $\alpha$ Ori and later Wilson et al. (1992) showed that the spots change in strength and number on timescales of months. Mira also showed large departures from symmetry in these measurements and it was of interest to determine whether such behaviour is typical of all red giants and super-giants. Recent work by Tuthill (1994) using the same techniques has established that hotspots containing a significant fraction of the light of the star occur in all the cases studied for which adequate angular resolution was available. Table 1 summarises the current statistics.

TABLE 1. Incidence of hot-spots on red supergiants and Mira variables

\begin{tabular}{lcccc}
\hline \multirow{4}{*}{ Supergiants } & & Epochs & No. of spots & \% flux in spots \\
& $\alpha$ Ori & 8 & $1-3$ & $11-29$ \\
& $\alpha$ Her & 2 & $1-2$ & $15-21$ \\
& $\alpha$ Sco & 1 & 1 & 5 \\
& $\mu$ Cep & 3 & 1 & $(9-30)$ \\
Miras & & & & \\
& o Cet & 4 & $1-3$ & $5-40$ \\
& R Leo & 1 & 1 & 13 \\
& $\chi$ Cyg & 1 & 1 & $7-9$ \\
R Cas & 2 & 2 & $25-30$ \\
\hline
\end{tabular}

\section{COAST}

The Cambridge Optical Aperture Synthesis Telescope was designed to be the smallest and simplest array of separate telescopes capable of providing high resolution imaging in the red and near inra-red. It has provision for baselines up to $100 \mathrm{~m}$, corresponding to about 1 milliarcsecond resolution. It consists of four independent telescopes, each comprising a $50-\mathrm{cm}$ siderostat flat feeding a 40-cm horizontal Cassegrain telescope with afocal optics. The $25-\mathrm{mm}$ emergent beams pass to a central laboratory through 


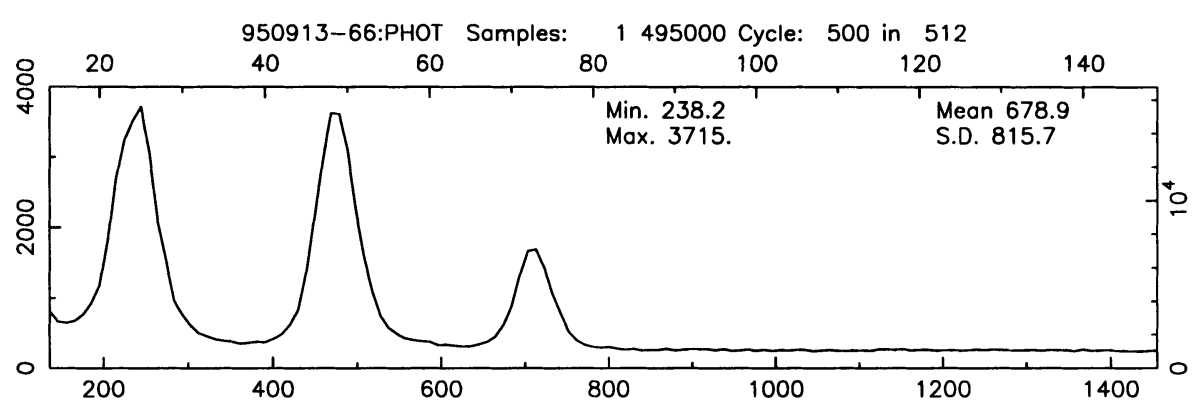

Figure 3. Power spectrum of fringes from three COAST baselines on Capella

white-painted alloy pipes in air at ambient pressure. The laboratory is covered to a depth of $1 \mathrm{~m}$ by earth to provide passive thermal stability for the beam-combining optics. Inside the laboratory the optical path of each telescope beam is corrected continuously for the differential path delays to the telescopes arising from earth rotation, using roof mirrors on railmounted trolleys. A fast-readout CCD detector provides tip-tilt signals to piezo-controlled mirrors on the telescopes for fast autoguiding.The beam mixing is performed using four 50:50 beam-splitter plates giving four collimated beams each of which contains one quarter of the light from each telescope. These are focused on to four separate avalanche photodiodes, operating in photon counting mode, as detectors. Interference takes place in the pupil plane and the fringes are detected by modulating the optical path in each arm of the interferometer, thus scanning the fringes past each detector. The critical setting is to equalise the paths such that fringes are detected on all baselines simultaneously. Different modulation of the path is made for each baseline, so that scan rates for the fringes differ.

Fig.3 shows the power spectrum of such fringe data from observations of Capella. Visibilities and closure phases from data of this type were used to make the first images with the COAST array in September 1995. The data were processed using the Caltech VLBI mapping package in an exactly similar manner to that used for radio data. It is notable that the only change necessary was that needed to display the frequency of observation correctly in the plots (see Fig.4). The images have a resolution of 20 milliarcsec, corresponding to the maximum baselines in the observations of $6.1 \mathrm{~m}$. They show the relative motion of the components of the binary over an interval of 15 days. The interest of the images is not principally for their astronomical value but as an important technical step; these are the first images from a separated-element phase-coherent array. The quality of the reconstruction is good with other features on the map less than 5 percent of the peak values. The position angles agree well with the most recent orbit determination. 

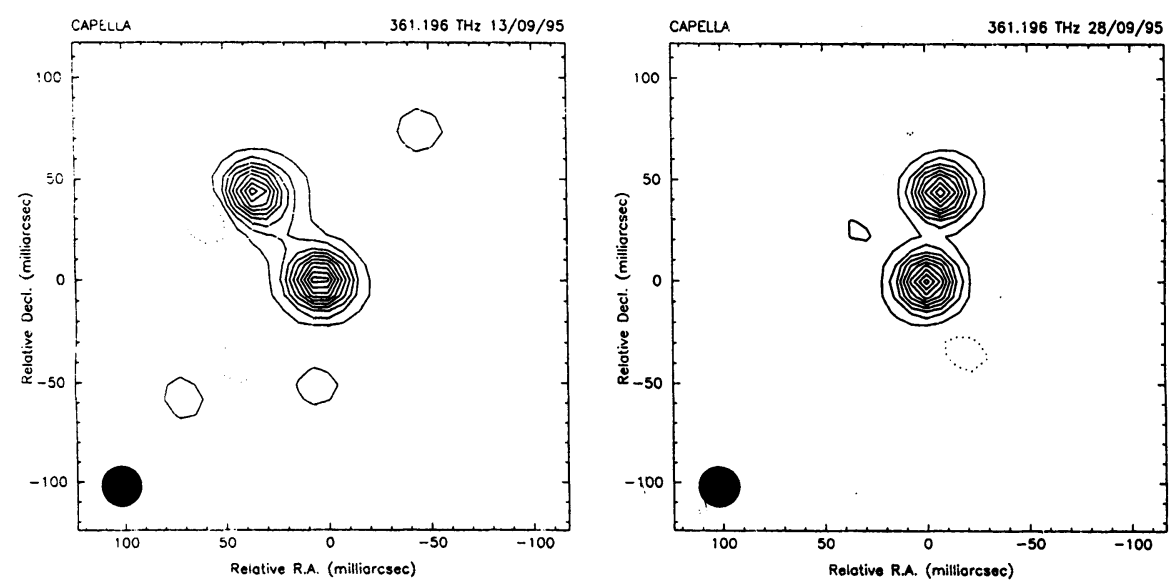

Figure 4. Images of Capella made with COAST on 950913 and 950928

A large range of programmes are now open for investigation. We plan, for instance, to follow up the studies of the hot-spots on red giants and supergiants to determine their sizes which are below the resolution limit in the non-redundant masking observations with $4 \mathrm{~m}$ telescopes. The quality of the images will depend on the ability to measure very low fringe visibilities or, equivalently, faint stars. The faintest limits we have achieved so far are a visibility $\mathrm{V}$ of 0.05 for $\alpha$ Her and a detection of fringes with good sinalto-noise ratio from one of the components of $61 \mathrm{Cyg}$ (fig.5) at $\mathrm{m}=+5.2$.

\section{FUTURE PROSPECTS}

The present situation in optical phase-coherent imaging corresponds roughly to that in radioastronomy in 1975 when the first simple images had been made using closure phase. The immediate prospects over the next five years are for further fairly simple images with improving resolutions down to about 1 milliarcsec from COAST and the other first generation arrays incorporating three or more telescopes, such as IOTA and the NPOI and in the slightly longer term KECK, CHARA and the VLTI. The character of the data obtained will be of fundamental importance in the design of the next generation of arrays and in estimating the limiting magnitude of the technique. The uncertainty at present concerns how small the visibilities are at baselines which lie beyond the first zero in the Fourier transform of the stellar brightness distribution. If they are very small, then future arrays will require large numbers of elements so that long baselines can be constructed by combining several short ones on which the visibilities are large. 


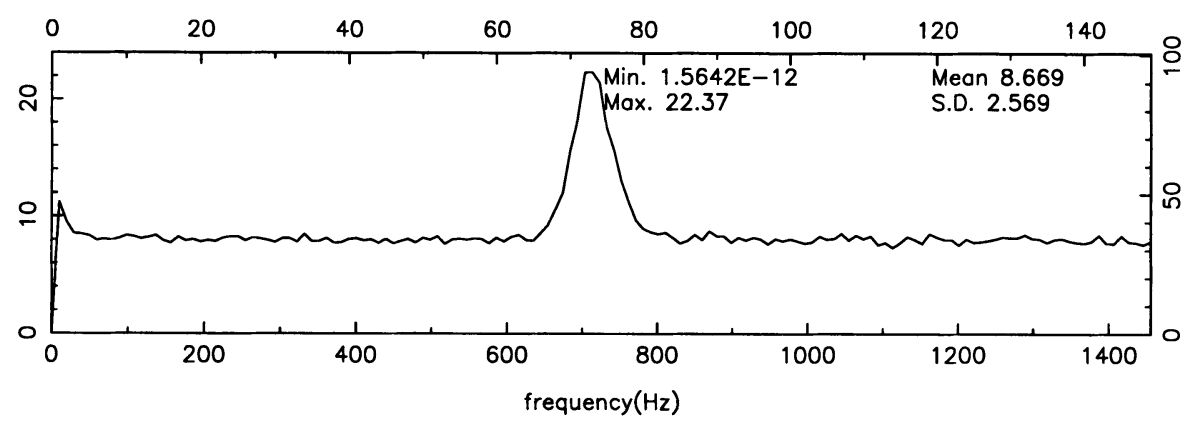

Figure 5. Power spectrum of fringes from $61 \mathrm{Cyg}$ with COAST

However the limiting magnitude will remain comparativly bright. Adaptive optics using natural guide stars offers little hope of improving that, since the limiting magnitude for adaptive optics to work on a given size of subpupil is closely similar to that for interferometry to be possible with the same size of of aperture. The use of laser guide stars could overcome this difficulty in principle, but the prospect of operating a large array of such stars is a daunting one.

An issue of particular interest for this meeting is how phase-coherent optical imaging will interface with the other methods which have been discussed here. My guess is that it is likely to complement rather than supplant them. For example, it is more suitable for slowly rotating stars and certainly less suitable, at present, for some of the objects of very small angular dimensions. But this is speculation. All one can say for sure is that we need every technique available.

\section{References}

Baldwin, J.E., Haniff, C.A., Mackay, C.D and Warner, P.J. (1986) Nature, Vol. no. 320 pp. 595-598

Buscher, D.F., Haniff, C.A.,Baldwin, J.E. and Warner, P.J. (1990) MNRAS, Vol. no. 245 pp. $7 \mathrm{p}-11 \mathrm{p}$

Jennison, R.C. (1958) MNRAS, Vol. no. 118 pp. 276-284

Labeyrie, A. (1975) ApJ, Vol. no. L71 pp. 71-75

Michelson, A.A. (1891) Nature, Vol. no. 45, pp. 160-161

O'Brien, P.A. (1953) MNRAS, Vol. no. 113 pp. 597-608

Rogers, A.E.E., Hinteregger, H.F., Whitney, A.R., Counselman, C.C., Shapiro, I.I. et al. (1974) ApJ, Vol. no. 193, pp. 293-301

Ryle, M. and Neville, A.C. (1962) MNRAS, Vol. no. 125 pp. 39-56

Tuthill, P.G. (1994) Ph.D' dissertation, Cambridge pp. 1-173

Wilson, R.W., Baldwin, J.E., Buscher, D.F. and Warner, P.J. (1992) MNRAS, Vol. no. 257 pp. 369-376 